\title{
Comparative study of serum proteomes in Legg-Calve-Perthes disease
}

\author{
Ruiyu Liu', Lihong Fan', Longbin Yin ${ }^{1}$, Kunzheng Wang ${ }^{1}$, Wusheng Miao², Qichun Song ${ }^{1}$, Xiaogian Dang ${ }^{1}$,
} Hang Gao ${ }^{3}$ and Chuanyi Bai ${ }^{1 *}$

\begin{abstract}
Background: Legg-Calve-Perthes Disease (LCPD) is an idiopathic osteonecrosis of the developing femoral head complicated by pain and disability of the hip joint. To date, the pathological mechanisms of LCPD are not well-known. This study screened the changes in serum protein expression in patients with LCPD.

Methods: Age- and sex-matched serum samples from 10 control subjects and 10 patients with LCPD were compared using the isobaric tags for relative and absolute quantification (iTRAQ) technique. Gene ontology analyses, KEGG pathway and functional network analyses were performed. Proteins of interest with large differences in expression, S100-A8, alpha-1-acid glycoprotein 1, haptoglobin and apolipoprotein E, were compared by western blotting.

Results: The disease/control ratios showed 26 proteins were significantly differentially expressed (all $p<0.05$ ). Including higher abundances of complement factor $\mathrm{H}(1.44)$, complement C4-B (1.45), isocitrate dehydrogenase [NAD] subunit alpha (2.7) alpha-1-acid glycoprotein 1 (1.87), heptoglobin (1.53) and Ig lambda-2 chain C regions (1.46), and lower levels of apolipoprotein E (0.50), apolipoprotein F (0.60), apolipoprotein C-III (0.69), S100-A8 (0.73), S100-A9 (0.75) and prothrombin (0.77) in LCPD than in controls. The alpha-1-acid glycoprotein 1 and haptoglobin increases, and apolipoprotein E and S100-A8 decreases were confirmed by western blot. KEGG pathway analysis revealed these proteins were related to the complement and coagulation cascades, Staphylococcus aureus infection, PPAR signaling, fat digestion and absorption, and vitamin digestion and absorption. Functional network analysis suggested that the proteins were involved in lipid regulation.
\end{abstract}

Conclusions: The complement and coagulation cascades, and abnormal lipid metabolism may be involved in the pathogenesis of LCPD.

Keywords: Legg-Calve-Perthes disease, Serum proteome, Isobaric tags for relative and absolute quantification, Lipid metabolism

\section{Background}

Legg-Calve-Perthes disease (LCPD) is an idiopathic osteonecrosis of the developing femoral head complicated by pain and disability of the hip joint. The disease affects childhood between 6 and 9 years of age. The estimated annual incidence ranges from 0.2 to 19.1 per 100,000 among children under 15 years [1]. It occurs 5 times more often in boys than in girls [2]. The main long-term problem with this disease is a permanent deformity of the femoral head, which increases the risk of

\footnotetext{
* Correspondence: baichuanyiyx@sina.com

'Department of Orthopaedic, the Second Hospital Affilicated to Medical College, Xi'an Jiaotong University, No.157, Xiwu Road, Xi'an, Shaanxi 710004, P. R China

Full list of author information is available at the end of the article
}

developing osteoarthritis in adults [3]. However, the underlying mechanisms and determinants of LCPD are still unclear including the proteins involved. This situation hampers effective treatment and etiological investigation.

Coagulation abnormalities [4-6], vascular interruptions of the blood supply to the proximal femur [7] and abnormal immunological reactivity [8] are all linked to the pathogenesis of LCPD. In addition, it has been suggested that leptin, a lipid regulator, might play an important role [9]. Another study showed abnormalities in the vascular structure and function in children with LCPD disease [10]. In experimental animal models, acute bone morphogenic protein 2 (BMP2) upregulation [11], increased vascular endothelial growth factor (VEGF) expression 
in the epiphyseal cartilage [12], and increased matrix mineralization [13] in the immature femoral head follow induction of ischemic osteonecrosis. These findings imply that multiple pathological reactions may be involved in the development of LCPD.

Comparative serum proteomic analysis has been used for years as a potent tool for understanding the pathology of many diseases, especially for those diseases, like LCPD, about which we have very limited knowledge. A similar strategy has been applied to osteonecrosis of the femoral head in adults $[14,15]$. Given that these two diseases occur in the same location and have similar symptoms the results of that study should also help us understand the pathology of LCPD. Isobaric tags for relative and absolute quantitation (iTRAQ), is a quantitative proteomic approach with relatively high throughput that allows for simultaneous identification and peptide quantification by measuring the peak intensities of reporter ions with tandem mass spectroscopy (MS/MS) that has been developed and utilized to identify biomarkers for various disease conditions [16, 17]. This chemical labeling method involves the stable incorporation of isotopes into an amine tagging reagent, which can then be reliably detected by mass spectrometry, thereby permitting comparative quantitation of various proteins. This method has been suggested to be suitable for the discovery of differentially expressed proteins in a wide range of body fluids and tissues, including serum and plasma $[17,18]$.

In this study, we performed a comparison of serum proteomes in child patients with LCPD using the bioinformation match iTRAQ method, to assess the simultaneous expression of several serum proteins. This allowed us to characterize the associations between differentially expressed proteins and the incidence of LCPD.

\section{Methods \\ Patients}

The study included a total of 20 healthy children and 20 patients with LCPD. All the patients and the gender/age matched healthy children were recruited between November 2011 and August 2012 from the second Hospital affiliated to Xian Jiaotong University (Xi'an, China). The diagnosis of LCPD was verified based upon the radiographic appearance and clinical features [19]. The recruited patients were diagnosed with the disease for the first time and they had accepted no treatment including agent, surgery or the other treatments (non-weight bearing, bed rest, cast, etc.) before their blood was sampled. Cases of multiple epiphyseal dysplasia, cerebral palsy, and developmental hip dysplasia were excluded owing to their known independent association with avascular necrosis of the hip. The severity of the disease was evaluated according to the Catterall staging system [19], there were four stage I patients, five stage II patients and one stage III patients. The healthy children, who had a physical examination before admission to our hospital, were randomly enrolled to serve as the control group in the same period by being age- and gender-matched. The recruitment criteria was as follows: the healthy children had no disease and bone disease history, with no previous or current symptoms related to the hip and a normal hip radiograph (if available) or no limitation of hip abduction and internal rotation (in children without hip radiographs). There were no significant differences in body weight and height between the two groups. ITRAQ analysis was performed for the first ten patients and ten healthy children (clinical characteristics are showed in Table 1). The blood sample of another 20 patients and the age and sex matched healthy children were used to performed west-blotting analysis (clinical characteristics are shown in Table 2).

This study was approved by the Ethical Committee of the Second Affiliated Hospital of Xian Jiaotong University, Xi'an, China. Signed informed-consent documents were obtained from all the study participants and their guardians.

\section{Protein Digestion and isobaric tags for relative and absolute quantification (iTRAQ) labeling}

Two milliliters of peripheral venous blood was obtained from each patient in the outpatient department, and processed to collect serum and then stored at $-80^{\circ} \mathrm{C}$ for further analysis. Fourteen high-abundance proteins in the serum pools were depleted using a multiple affinity removal system (Agilent Human 14, Agilent Technologies, USA) following the manufacturer's protocol. A 5 KDa ultrafiltration tube (Sartorius) was used for desalination and concentration. The protein content of the depleted serum was determined by the Bradford assay (Bio-Rad protein assay) according to the manufacturer's instructions.

Protein digestion was performed according to the Filter aided sample preparation (FASP) procedure described by Wisniewski et al. [16]. The resulting peptide mixture was labeled using the 4-plex iTRAQ reagent (Applied Biosystems). Briefly, $200 \mu \mathrm{g}$ of peptides from each sample were added to $30 \mu$ of STD buffer (4\% sodium dodecyl sulfate (SDS), $100 \mathrm{mM}$ dithiothreitol (DTT), $150 \mathrm{mM}$ Tris- $\mathrm{HCl}$ $\mathrm{pH}$ 8.0). The detergent, DTT and other low-molecularweight components were removed using UA buffer $(8 \mathrm{M}$

Table 1 Clinical characteristics in two groups for iTRAQ analysis

\begin{tabular}{lll}
\hline Group & Patient group & Healthy group \\
\hline Sex (female /male) & $1 / 9$ & $1 / 9$ \\
Age (Years) & $5.4 \pm 1.9$ & $5.5 \pm 2.0$ \\
Height $(\mathrm{cm})$ & $111.5 \pm 14.9$ & $109.6 \pm 15.1$ \\
Weight $(\mathrm{kg})$ & $20.5 \pm 5.1$ & $20.9 \pm 4.8$ \\
\hline
\end{tabular}


Table 2 Clinical characteristics in two groups for West-blotting verification

\begin{tabular}{lll}
\hline Group & Patient group & Healthy group \\
\hline Sex (female /male) & $3 / 17$ & $3 / 17$ \\
Age (Years) & $5.6 \pm 2.1$ & $5.8 \pm 2.3$ \\
Height $(\mathrm{cm})$ & $113.5 \pm 12.3$ & $108.6 \pm 16.3$ \\
Weight $(\mathrm{kg})$ & $22.5 \pm 5.4$ & $21.9 \pm 5.0$ \\
\hline
\end{tabular}

Urea, $150 \mathrm{mM}$ Tris- $\mathrm{HCl} \mathrm{pH}$ 8.0) by repeated ultrafiltration (Microcon units, $30 \mathrm{kD}$ ). Then $100 \mu \mathrm{l} 0.05 \mathrm{M}$ iodoacetamide in UA buffer was added as a blocking agent and the samples were incubated for $20 \mathrm{~min}$ in darkness. The filters were washed with $100 \mu \mathrm{l}$ of UA buffer three times and then $100 \mu \mathrm{l}$ DS buffer (50 mM triethylammoniumbicarbonate at $\mathrm{pH}$ 8.5) twice. Finally, the protein suspensions were digested with $2 \mu \mathrm{g}$ of trypsin (Promega, Madison, USA) in $40 \mu \mathrm{l}$ of DS buffer overnight at $37^{\circ} \mathrm{C}$, and the resulting peptides were collected as a filtrate. The peptide content was estimated by UV light spectral density at $280 \mathrm{~nm}$ using an extinction coefficient of 1.1 of $0.1 \%(\mathrm{~g} / \mathrm{l})$ solution that was calculated on the basis of the frequency of tryptophan and tyrosine in vertebrate proteins $[20,21]$.

For labeling, each iTRAQ reagent was dissolved in $70 \mu \mathrm{l}$ of ethanol and added to the respective peptide mixture. The samples were labeled as Serum1-114, Serum2-115, Serum3-116, and Serum4-117, and were multiplexed and vacuum dried.

\section{Mass Spectrum (MS) analyses}

The peptide mixture was desalted on C18 Cartridges (Empore $^{\mathrm{Tm}}$ SPE Cartridges C18, standard density, bed I.D. $7 \mathrm{~mm}$, volume $3 \mathrm{ml}$, Sigma, St. Louis, USA), then concentrated by vacuum centrifugation and reconstituted in $40 \mu \mathrm{l}$ of $0.1 \%(\mathrm{v} / \mathrm{v})$ trifluoroacetic acid. MS experiments were performed on a $\mathrm{Q}$ Exactive mass spectrometer that was coupled to Easy nLC (Proxeon Biosystems, now Thermo Fisher Scientific). $10 \mu \mathrm{l}$ of the sample was injected for nanoLC-MS/MS analysis. The peptide mixture $(5 \mu \mathrm{g})$ was loaded onto a C18-reversed phase column $(15 \mathrm{~cm}$ long, $75 \mu \mathrm{m}$ inner diameter) packed in-house with RPC18 $5 \mu \mathrm{m}$ resin in buffer A (0.1 \% Formic acid) and separated with a linear gradient of buffer B (80\% acetonitrile and $0.1 \%$ Formic acid) at a flow rate of $250 \mathrm{nl} / \mathrm{min}$ controlled by IntelliFlow technology over $240 \mathrm{mins}$. MS data was acquired using a data-dependent top 10 method dynamically choosing the most abundant precursor ions from the survey scan $(300-1800 \mathrm{~m} / \mathrm{z})$ for HCD (higher energy collisional dissociation) fragmentation. Determination of the target value was based on predictive automatic gain control (pAGC). Dynamic exclusion duration was $60 \mathrm{~s}$. Survey scans were acquired at a resolution of 70,000 at $\mathrm{m} / \mathrm{z} 200$ and resolution for HCD spectra was set to 17,500 at $\mathrm{m} / \mathrm{z} 200$. Normalized collision energy was $30 \mathrm{eV}$ and the underfill ratio, which specifies the minimum percentage of the target value likely to be reached at maximum fill time, was defined as $0.1 \%$. The instrument was run with peptide recognition mode enabled.

\section{Protein identification}

MS/MS spectra were searched using MASCOT engine (Matrix Science, London, UK; version 2.2) embedded into Proteome Discoverer 1.3 (Thermo Electron, San Jose, CA.) against UniProt Human database (133549 sequences, downloaded on March 3rd, 2013) and the decoy database. For protein identification, the following options were used. Peptide mass tolerance $=20 \mathrm{ppm}$, MS $/$ MS tolerance $=0.1 \mathrm{Da}$, Enzyme $=$ Trypsin, Missed cleavage $=2$, Fixed modification: Carbamidomethyl $(C)$, iTRAQ4plex (K), iTRAQ4plex (N-term).

\section{Gene ontology analyses}

The gene ontology (GO) method is a major bioinformatics initiative with the aim of standardizing the representation of protein attributes across species and databases. The online system provides a controlled vocabulary of terms for describing gene product characteristics and gene product annotation data from GO consortium members, as well as tools to access and process this data. Each protein is characterized in terms of three ontologies: molecular function, cellular component and the involved biological process. Using the GO database (http://www.geneontology.org/) and Onto-Express analysis [18], the involved genes were classified in order to gain an overall picture of potential functions of the differentially expressed genes in this study.

\section{KEGG pathway analyses}

The orthology of the proteins was investigated using the Kyoto encyclopedia of genes and genomes (KEGG) database, by adopting a web-based server called KAAS (KEGG Automatic Annotation Server: http://www.genome.jp/ $\mathrm{kegg} / \mathrm{kaas} /$ ). The proteins were annotated with the KEGG orthology (KO) identifiers, or the $\mathrm{K}$ numbers, based on the best hit information using Smith-Waterman scores as well as by the manual curation. Each $\mathrm{K}$ number represents an ortholog group of genes, and it is directly linked to an object in the KEGG pathway map or the BRITE functional hierarchy. The method is based on sequence similarities, bi-directional best hit information and some heuristics, and has achieved a high degree of accuracy when compared with the manually curated KEGG GENES database.

\section{Functional network analyses}

To investigate the direct (physical) and/or indirect (functional) interactions among the identified genes, we further utilized the search tool for the retrieval of interacting genes 
(STRING) database (http://string.embl.de/) to analyze the functional network. The STRING database provided a score for each gene-gene interaction, which is computed as the joint probability of the probabilities from the different evidence channels (protein interaction, fusion, co-expression, text mining, etc.), correcting for the probability of randomly observing an interaction [22]. A high database score meant that there were high experimental or predicted evidence for gene-gene functional interaction. Therefore a functional associated network was constructed based on the differential expression profile in the present study.

\section{Western blotting analysis}

Commercial antibodies used for western blotting validation experiments were as follows: haptoglobin (HP) (31083) (Signalway Antibody LLC, Baltimore, USA), S100A8 (ab92331), and orosomucoid 1 (ORM1) (ab133642), apolipoprotein E (APOE) (ab52607) and actin monoclonal antibodies (all from Abcam, Cambridge, MA, USA). Processed serum from another 30 adolescents (15 LCPD patients and 15 age and sex matched healthy adolescents) that had undergone depletion of high-abundance proteins was used for western blotting analysis. SDS polyacrylamide gel electrophoresis (SDS-PAGE) was performed on $12-15 \%$ acrylamide gels with $60 \mu \mathrm{g}$ protein per lane, and proteins were electrophoretically transferred onto nitrocellulose membranes (Millipore, Billerica, MA, USA). Membranes were blocked with blocking buffer purchased from LICOR (Lincoln, NE, USA) and then incubated overnight at $4{ }^{\circ} \mathrm{C}$ with primary antibody. The dilutions were performed as suggested by the manufacturers. The membranes were washed with phosphate buffered saline (PBS) followed by incubation with a horseradish peroxidaseconjugated secondary antibody (Santa Cruz Biotechnology, Inc., Santa Cruz, CA, USA) at $37^{\circ} \mathrm{C}$ for 2 hours, and then chemiluminescence detection by ECL plus. Captured images were analyzed using a LAS4000 (Fuji Film) and Multi Gaugev2.0 (Fuji Film). For quantification of western blotting data, each protein signal was quantitated and normalized by the internal protein actin intensity.

\section{Statistical analysis}

The statistical significance of the differential expression levels on proteins was tested by $t$-test. A protein with a

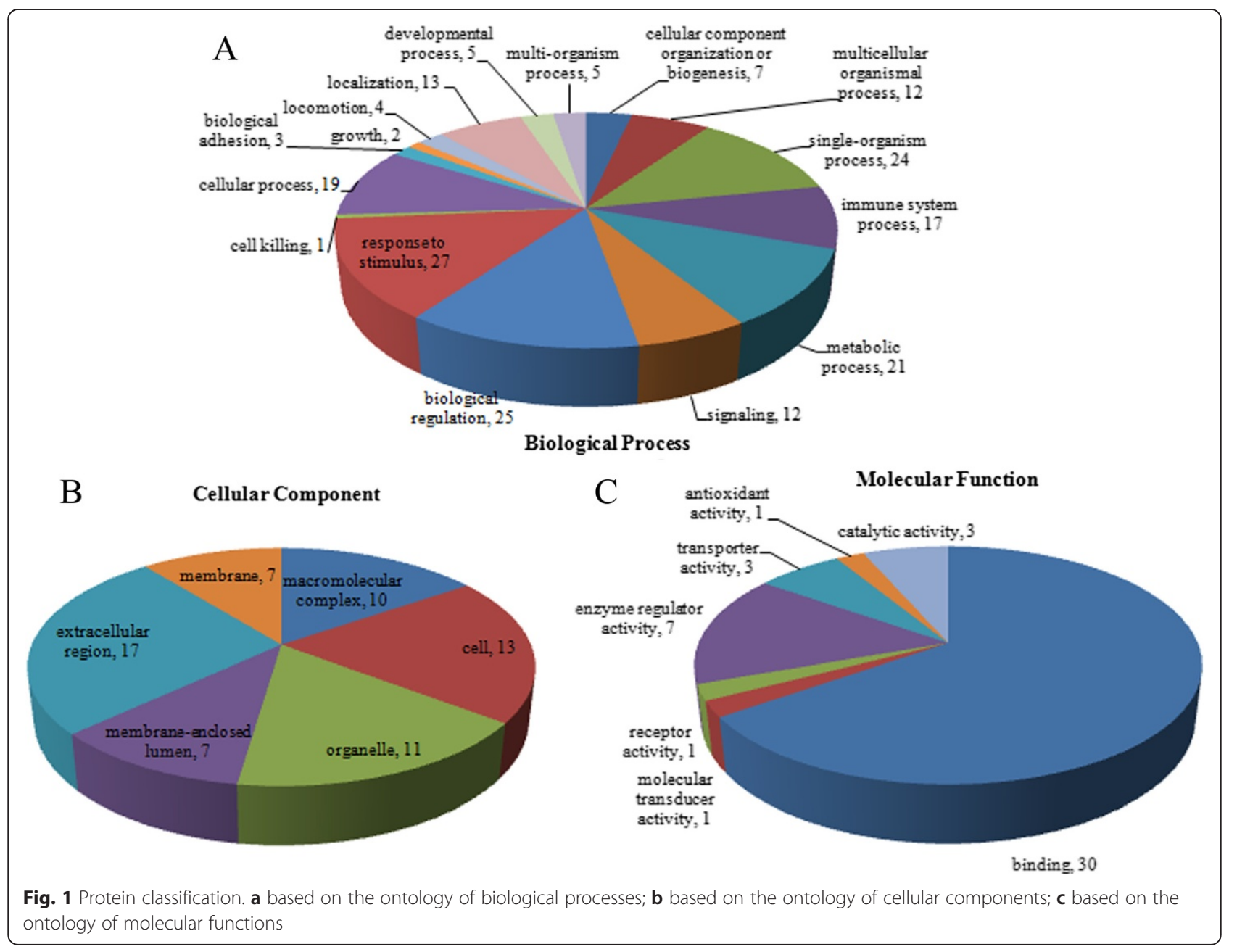


disease/healthy control ratio $>1.2$ and $p<0.05$ was considered as significant. The method of false discovery rate (FDR) was used for addressing the multiple-testing adjustment, and adjusted $p<0.01$ was used as the significant criterion.

\section{Results}

\section{Protein identification}

We identified 223 protein groups from a total of 2803 unique peptides. All the identified proteins were classified using the GO annotation (http://david.abcc.ncifcrf.gov/ home.jsp) and further categorized into three functional groups: biological processes (Fig. 1a), cellular components (Fig. 1b) and molecular functions (Fig. 1c).

The GO analyses showed that the most common molecular function was binding activity, which included ion binding, protein binding, carbohydrate binding, pattern binding, cell surface binding, and lipid binding. Another major functional category was enzyme regulation. The most frequently encountered biological processes were biological regulation, response to stress, localization, and establishment of localization.

\section{Differentially expressed proteins}

There was a significant difference of expression of 26 proteins that had specific functions between the disease and control groups (shown in Table 3). Complement activation related proteins, complement C4-B and complement factor $\mathrm{H}$, were more highly expressed in the disease group. However, the cartilage metabolism related protein S100A8/S100-A9 was reduced in the disease group. Furthermore, we found that the immune response related protein, Ig kappa chain $C$ region and Ig lambda- 2 chain $C$ region, had significant increased expression in LCPD. And prothrombin, which has a close relationship with coagulation, was lower in the disease group.

The KEGG pathway analysis showed that the complement and coagulation cascades were the major pathways in the study. In addition pathways related to Staphylococcus aureus infection, peroxisome proliferator-activated

Table 3 Differentially Expressed Proteins in LCPD and Control Groups

\begin{tabular}{|c|c|c|c|c|}
\hline Accession no. & Protein description & Gene name & Disease/control Ratio & $p$-value \\
\hline Q8TCZ8 & Apolipoprotein E & APOE & 0.50 & $<0.001$ \\
\hline F5GXS5 & Apolipoprotein F & APOF & 0.60 & $<0.001$ \\
\hline B7Z539 & Inter-alpha-trypsin inhibitor heavy chain $\mathrm{H} 1$ & $\mathrm{ITIH} 1$ & 0.67 & $<0.001$ \\
\hline P02656 & Apolipoprotein C-III & APOC3 & 0.69 & $<0.001$ \\
\hline P05109 & S100-A8 & S100A8 & 0.73 & 0.004 \\
\hline P01860 & Ig gamma-3 chain $C$ region & IGHG3 & 0.74 & 0.006 \\
\hline Q59HB3 & Apolipoprotein B & APOB & 0.74 & 0.007 \\
\hline B2R4M6 & S100-A9 & S100A9 & 0.75 & 0.008 \\
\hline P02655 & Apolipoprotein C-II & APOC2 & 0.76 & 0.010 \\
\hline P00734 & Prothrombin & F2 & 0.77 & 0.017 \\
\hline B2R701 & Peptidase inhibitor 16 & PI16 & 0.79 & 0.027 \\
\hline P11226 & Mannose-binding protein C & MBL2 & 0.79 & 0.030 \\
\hline B7Z544 & Inter-alpha-trypsin inhibitor heavy chain $\mathrm{H} 4$ & $\mathrm{ITIH} 4$ & 0.80 & 0.040 \\
\hline P36980 & Complement factor H-related protein 2 & CFHR2 & 0.80 & 0.470 \\
\hline B3KWB5 & Alpha-1B-glycoprotein & $\mathrm{A} 1 \mathrm{BG}$ & 1.288 & 0.033 \\
\hline P01859 & Ig gamma-2 chain $C$ region & IGHG2 & 1.315 & 0.020 \\
\hline A8K5T0 & Complement factor $\mathrm{H}$ & $\mathrm{CFH}$ & 1.44 & 0.002 \\
\hline B7Z1F8 & Complement C4-B & $\mathrm{C} 4 \mathrm{~B}$ & 1.45 & 0.001 \\
\hline POCG05 & Ig lambda-2 chain $C$ regions & IGLC2 & 1.46 & 0.001 \\
\hline A2J1M4 & Rheumatoid factor RF-ET7 (Fragment) & & 1.46 & 0.001 \\
\hline P00738 & Haptoglobin & $\mathrm{HP}$ & 1.53 & $<0.001$ \\
\hline P01834 & Ig kappa chain $C$ region & IGKC & 1.60 & $<0.001$ \\
\hline P02763 & Alpha-1-acid glycoprotein 1 & ORM1 & 1.87 & $<0.001$ \\
\hline P01009 & Alpha-1-antitrypsin & SERPINA1 & 1.93 & $<0.001$ \\
\hline P02647 & Apolipoprotein A-I & APOA1 & 2.0 & $<0.001$ \\
\hline HOYLI6 & Isocitrate dehydrogenase [NAD] subunit alpha, mitochondrial & $\mathrm{IDH} 3 \mathrm{~A}$ & 2.7 & $<0.001$ \\
\hline
\end{tabular}


receptor (PPAR) signaling, fat digestion and absorption, and vitamin digestion and absorption were also identified (shown in Fig. 2).

To further analyze the 26 differently expressed proteins using STRING, a functional associated network was constructed (shown in Fig. 3). This indicated that APOE was employed in the central role in the LCPD network.

\section{Western blotting validation}

Western blot analysis of four differentially expressed proteins (S100-A8, haptoglobin, alpha-1-acid glycoprotein 1 and apolipoprotein E) was used to verify our mass spectrometry results. They were selected for investigation based on their interesting biological functions and high foldchanges, as well as the availability of commercial antibodies. ORM1 and HP were increased in patients with LCPD, APOE and S100-A8 were decreased in patients with LCPD (shown in Fig. 4). Thus, western blotting was consistent with the results of the mass spectrometry analysis.

\section{Discussion}

In this study, we compared serum proteome expression in LCPD in a Chinese Hans population, and found some prominently differentially expressed proteins in the LCPD patients compared with the healthy controls. The differential serum proteomes provide new-insights into the systemic expression of many reactions that may have occurred in the tissues of patients with LCPD.

Abnormal coagulation has been shown to be closely related with the occurrence of LCPD [4-6]. In our study we found that prothrombin, which is important for blood

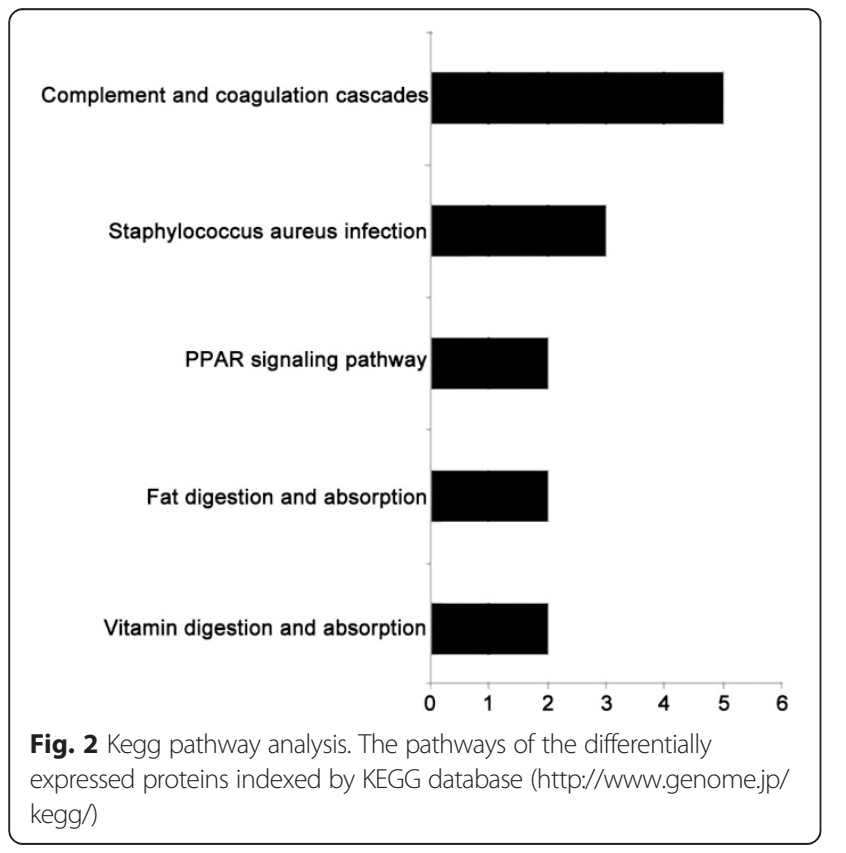

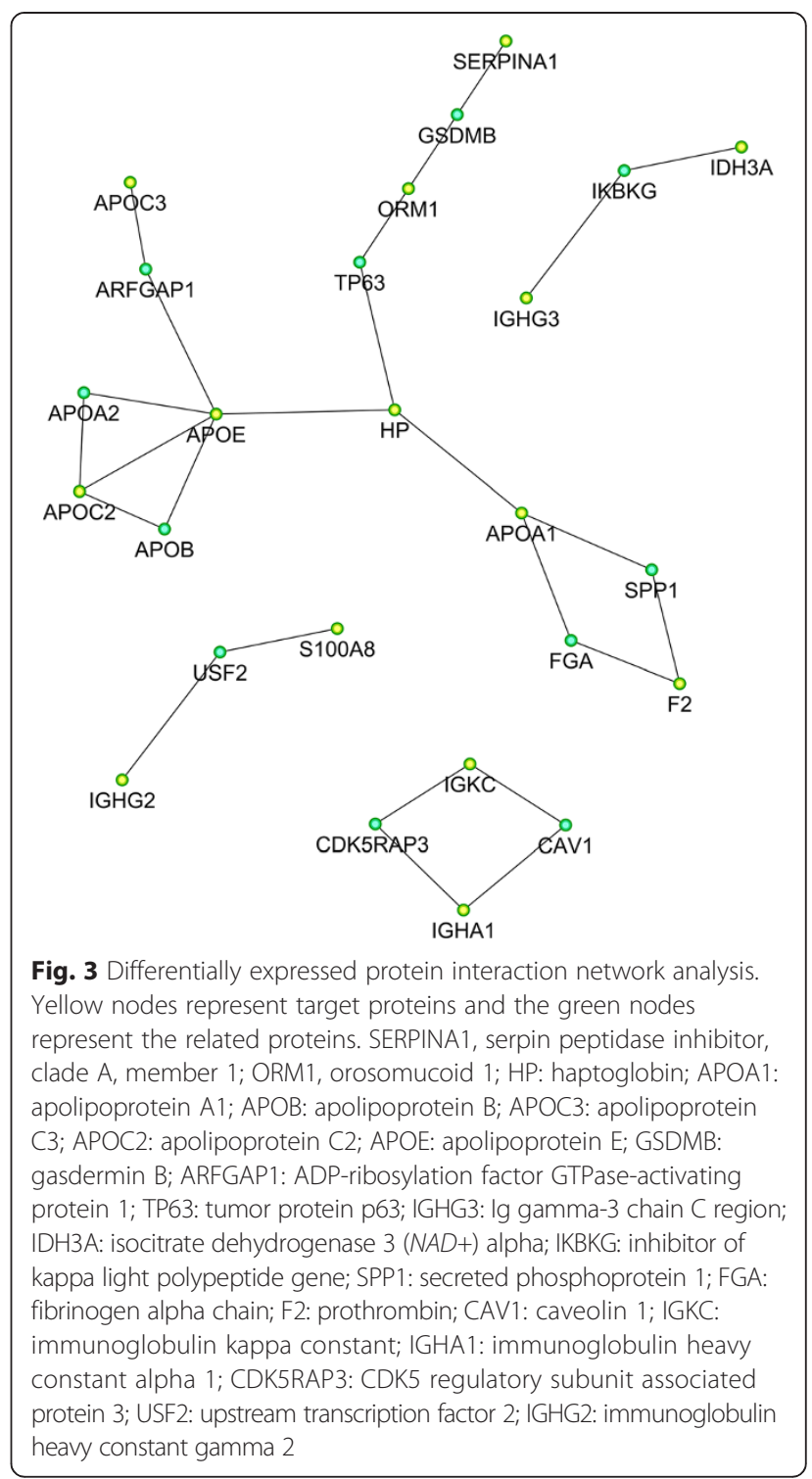

coagulation, had a different expression level, which is consistent with the previous studies [4-6].

A recent study reported that abnormalities of vascular structure and function occur in children with LCPD [10]. We found nicotinamide adenine dinucleotide (NAD) expression was suppressed in LCPD. Activation of NAD can prevent atherosclerosis and arterial restenosis after vascular injury by suppressing vascular smooth muscle cell proliferation [23].

An immune response is involved in the pathogenesis of many diseases, including femoral head necrosis in adult patients [24]. A previous study showed that genetic variants of interleukin- 6 were related with the occurrence of the LCPD $[8,25]$. We observed that immune response related proteins were upregulated in LCPD, revealing that an immune response may be involved in this disease. These proteins may also be involved in complement activation. 

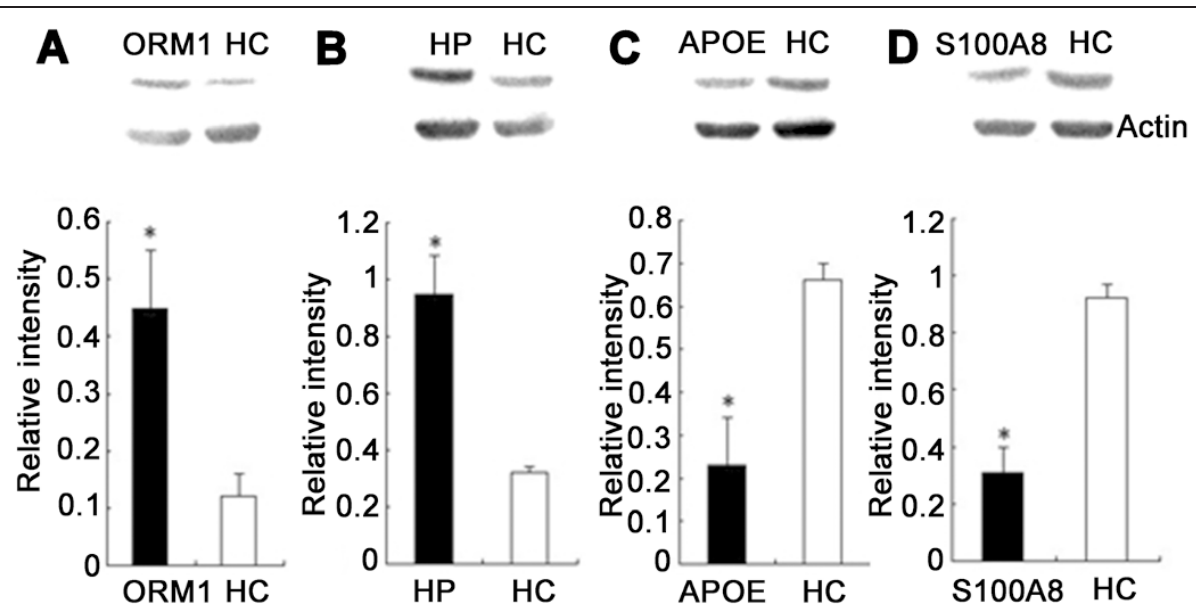

Fig. 4 Western blot analysis of four differentially expressed proteins. Relative intensities of the positively identified proteins are shown by the histograms. Patients with Legg-Calve-Pathes disease (LCPD) displayed higher levels of a orosomucoid 1 (ORM1) and $\mathbf{b}$ haptoglobin (HP), and lower levels of $\mathbf{c}$ apolioprotein $\mathrm{E}(A P O E)$ and $\mathbf{d}$ S100-A8 than healthy controls (HC). The relative density was calculated by dividing the density of matched spot by the density of all the matched spots in the respective gel. *indicates significant difference from the healthy volunteer group, $p<0.05$

The LCPD patients showed higher abundance of complement $\mathrm{C} 4-\mathrm{B}$ and complement factor $\mathrm{H}$, which are involved in prohibition of complement activation. Complement activation is supposed to involved in osteoarthritis pathogenesis [26, 27] and adult femoral head necrosis [15]. Therefore, we supposed that cartilage destruction was involved in the pathogenesis of LCPD [28-30]. Very few studies have focused on the pathological role of complement factors in regulating LCPD. Complement factors reportedly control the clearance of necrotic or apoptotic cells in tissue remodeling. The potent biological role of complement factor $\mathrm{H}$ in LCPD is unclear. In our study, changes in the abundances of complement factor $\mathrm{C}$ presumably indicated disturbances in the homeostasis of inflammatory, necrotic, or apoptotic reactions in LCPD.

S100 proteins are low molecular weight (9 to $14 \mathrm{kDa}$ ) intracellular calcium-binding proteins that control key cellular pathways. Chondrocytes and osteoblasts have been shown to express S100-A8 and S100-A9. Both S100-A8 and S100-A9 may contribute to calcification of the cartilage matrix and its replacement with trabecular bone, and redox regulation during bone resorption [31]. They have a possible role in cartilage repair or inflammation-induced degradation [32]. Protein S100-A8, is supposed to be involved in the pathology of osteoarthritis [33, 34], and to have a close relationship with rheumatoid arthritis. Serum levels of S100-A8/S100-A9 were found to correlate better with disease activity and joint destruction in various inflammatory arthritis than classical markers of inflammation [35, 36]. However, both S100-A8 and S100-A9 were decreased in the serum of LCPD patients, showing that LCPD has a different pathogenesis to osteoarthritis.
The functional network of the differently expressed proteins showed that 10 proteins, including serpin peptidase inhibitor, clade A, ORM1, HP, transferrin, APOA1, APOB, APOC3, APOC3, APOE, and APOF, were closely connected by relatively thick lines. Based on the KEGG analysis, these proteins are functionally related to lipid metabolism. Apolipoprotein genes have been repeatedly associated with osteonecrosis [37, 38]. The availability of multiple lines of evidence further strengthens the validity of the relationship between lipid metabolism and LCPD.

Actually, abnormal lipid metabolism has been considered to be an indicator of clinical risk for osteonecrosis and LCPD [39, 40]. It has been shown that lipid metabolism disorders are related to the occurrence of the disease [41]. Furthermore, a recent study reported that leptin played an important role in LCPD pathogenesis [9]. Genetic polymorphisms involved in lipid metabolism were also associated with osteonecrosis of the femoral head in Chinese patients [42]. Taken together, these results suggest that abnormal lipid metabolism may play an important role in the pathology of LCPD.

This study has some limitations. We performed the proteomic analysis on two quite small study populations, although we did verify four differentially expressed genes in a larger population. Further analysis of these results in larger study populations from different ethnic groups would provide more weight to the results. We also did not perform any investigation into the mechanisms suggested by this study. The findings of the present study are the first step in elucidating the proteomic regulation of LCPD. Further Studies will functionally characterize the biological role of the candidate proteins in modulating the 
development of LCPD. Those details will have to be revealed in later studies.

\section{Conclusion}

Our serum proteomic observations suggest that multiple pathological reactions may occur in the development of LCPD. We have characterized the expression profile and, by extrapolation, defined the functional relationship of a specific set of proteins that were differentially expressed between the LCPD and control groups. The bioinformatics analyses especially highlighted the importance of abnormal lipid metabolism to the occurrence of LCPD.

\section{Abbreviations \\ LCPD: Legg-Calve-Perthes Disease; iTRAQ: Isobaric tags for relative and absolute quantification; SDS: Sodium dodecyl sulfate; DTT: Dithiothreitol; pAGC: Predictive automatic gain control; FASP: Filter aided sample preparation; FDR: False discovery rate; GO: Gene ontology; STRING: Search Tool for the Retrieval of Interacting Genes; SERPINA1: Serpin peptidase inhibitor, clade A, member 1; ORM1: Orosomucoid 1; ALB: Albumin; HP: Haptoglobin; TF: Transferrin; APOA1: Apolipoprotein A1; APOB: Apolipoprotein B; APOC3: Apolipoprotein C3; APOC2: Apolipoprotein C2; APOE: Apolipoprotein E; APO: Apolipoprotein F; KEGG: Kyoto encyclopedia of genes and genomes; NAD: Nicotinamide adenine dinucleotide.}

\section{Competing interests}

The authors declare that they have no competing interests.

\section{Authors' contributions}

RYL, CYB, XQD and KZW Conceived and designed the experiments; LBY, HG and QCS performed the experiments and collected the serum sample; LHF, HG and QCS analyzed the data; RYL, CYB, LHF and LBY wrote the paper. All authors read and approved the final manuscript.

\section{Authors' information}

Not applicable.

\section{Acknowledgements}

This study was supported by the National Natural Science Foundation of China (grant No. 81101337, and grant No.81101363), the New Teacher Fund of the Ministry of Education (grant No. 20110201120062). Written informed consent was obtained from the patients and their guardians for publication of this manuscript and accompanying images. We thank Shanghai Institutes for Biological Sciences, Chinese Academy of Sciences, for their technical support for the proteome analysis.

\section{Author details}

'Department of Orthopaedic, the Second Hospital Affilicated to Medical College, Xi'an Jiaotong University, No.157, Xiwu Road, Xi'an, Shaanxi 710004 P. R China. 'Department of Pediatric Orthopaedic, Hong-Hui Hospital, Medical College of Xi'an Jiaotong University, No. 76 Nanguo Road, Nanshao Men, Beilin District, Xi'an 710054, P. R China. ${ }^{3}$ Research Center for Proteome Analysis, Shanghai Institutes for Biological5 Sciences, Chinese Academy of Sciences, Shanghai, P. R China.

Received: 24 February 2015 Accepted: 21 September 2015 Published online: 05 October 2015

\section{References}

1. Perry DC, Machin DM, Pope D, Bruce CE, Dangerfield P, Platt MJ, et al. Racial and geographic factors in the incidence of Legg-Calvé-Perthes' disease: a systematic review. Am J Epidemiol. 2012;175(3):159-66.

2. Guille JT, Lipton GE, Szöke G, Bowen JR, Harcke HT, Glutting JJ. Legg-CalvéPerthes disease in girls. A comparison of the results with those seen in boys. J Bone Joint Surg Am. 1998;80(9):1256-63.

3. Shah H. Perthes disease: evaluation and management. Orthop Clin North Am. 2014;45(1):87-97.
4. Aksoy MC, Aksoy DY, Haznedaroglu IC, Sayinalp N, Kirazli S, Alpaslan M. Thrombomodulin and GFC levels in Legg-Calve-Perthes disease. Hematology. 2008;13(6):324-8.

5. Vosmaer A, Pereira RR, Koenderman JS, Rosendaal FR, Cannegieter SC. Coagulation abnormalities in Legg-Calvé-Perthes disease. J Bone Joint Surg Am. 2010;92(1):121-8.

6. Balasa W, Gruppo RA, Glueck CJ, Wang P, Roy DR, Wall EJ, et al. Legg-CalvePerthes disease and thrombophilia. J Bone Joint Surg Am. 2004;86-A(12):2642-7.

7. Atsumi T, Yamano K, Muraki M, Yoshihara S, Kajihara T. The blood supply of the lateral epiphyseal arteries in Perthes' disease. J Bone Joint Surg Br. 2000;82(3):392-8

8. Gaikko GV, Goncharova LD, Zolotukhin SE, Donchenko LI. [Mechanism of early metabolism disorders and immunological reactivity in children with Perthes disease]. Lik Sprava. 2001;1:56-61. Russian.

9. Lee JH, Zhou L, Kwon KS, Lee D, Park BH, Kim JR. Role of leptin in leggcalvé-perthes disease. J Orthop Res. 2013;31(10):1605-10.

10. Perry DC, Green DJ, Bruce CE, Pope D, Dangerfield P, Platt MJ, et al. Abnormalities of vascular structure and function in children with Perthes disease. Pediatrics. 2012;130(1):e126-31.

11. Kamiya N, Shafer S, Oxendine I, Mortlock DP, Chandler RL, Oxburgh L, et al. Acute BMP2 upregulation following induction of ischemic osteonecrosis in immature femoral head. Bone. 2013;53(1):239-47.

12. Kim HK, Bian H, Randall T, Garces A, Gerstenfeld LC, Einhorn TA. Increased VEGF expression in the epiphyseal cartilage after ischemic necrosis of the capital femoral epiphysis. J Bone Miner Res. 2004;19(12):2041-8.

13. Hofstaetter JG, Roschger P, Klaushofer K, Kim HK. Increased matrix mineralization in the immature femoral head following ischemic osteonecrosis. Bone. 2010;46(2):379-85.

14. Zhang H, Zhang L, Wang J, Ma Y, Zhang J, Mo F, et al. Proteomic analysis of bone tissues of patients with osteonecrosis of the femoral head. OMICS. 2009;13(6):453-66.

15. Wu RW, Wang FS, Ko JY, Wang CJ, Wu SL. Comparative serum proteome expression of osteonecrosis of the femoral head in adults. Bone. 2008;43(3):561-6.

16. Wiśniewski JR, Zougman A, Mann M. Combination of FASP and StageTip-based fractionation allows in-depth analysis of the hippocampal membrane proteome. J Proteome Res. 2009;8(12):5674-8.

17. Carbon S, Ireland A, Mungall CJ, Shu S, Marshall B, Lewis S, et al. Web Presence Working Group. AmiGO: online access to ontology and annotation data. Bioinformatics. 2009;25(2):288-9.

18. Khatri P, Bhavsar P, Bawa G, Draghici S. Onto-Tools: an ensemble of webaccessible, ontology-based tools for the functional design and interpretation of high-throughput gene expression experiments. Nucleic Acids Res. 2004;1(32):W449-56. Web Server issue.

19. Catterall A. The natural history of Perthes' disease. J Bone Joint Surg Br. 1971;53(1):37-53.

20. Yang $Y, B u$ D, Zhao $X$, Sun $P$, Wang J, Zhou L. Proteomic analysis of cow yak, buffalo, goat and camel milk whey proteins: quantitative differential expression patterns. J Proteome Res. 2013;512(4):1660-7.

21. Unwin RD, Griffiths JR, Whetton AD. Simultaneous analysis of relative protein expression levels across multiple samples using iTRAQ isobaric tags with 2D nano LC-MS/MS. Nat Protoc. 2010;5(9):1574-82.

22. von Mering $C$, Jensen $\sqcup$, Snel B, Hooper SD, Krupp M, Foglierini M, et al. STRING known and predicted protein-protein associations, integrated and transferred across organisms. Nucleic Acids Res. 2005;1(33):D433-7. Database issue.

23. Kim SY, Jeoung NH, Oh CJ, Choi YK, Lee HJ, Kim HJ, et al. Activation of NAD (P) H:quinone oxidoreductase 1 prevents arterial restenosis by suppressing vascular smooth muscle cell proliferation. Circ Res. 2009;104(7):842-50.

24. Okazaki S, Nishitani Y, Nagoya S, Kaya M, Yamashita T, Matsumoto H. Femoral head osteonecrosis can be caused by disruption of the systemic immune response via the toll-like receptor 4 signalling pathway. Rheumatology (Oxford). 2009;48(3):227-32.

25. Srzentić S, Spasovski V, Spasovski D, Zivković Z, Matanović D, Bascarević Z, et al. Association of gene variants in TLR4 and IL- 6 genes with Perthes disease. Srp Arh Celok Lek. 2014;142(7-8):450-6.

26. Wang Q, Rozelle AL, Lepus CM, Scanzello CR, Song JJ, Larsen DM, et al. Identification of a central role for complement in osteoarthritis. Nat Med. 2011;17(12):1674-9.

27. John T, Stahel PF, Morgan SJ, Schulze-Tanzil G. Impact of the complement cascade on posttraumatic cartilage inflammation and degradation. Histol Histopathol. 2007;22(7):781-90. 
28. Zilkens $C$, Holstein A, Bittersohl B, Jäger M, Haamberg T, Miese F, et al. Delayed gadolinium-enhanced magnetic resonance imaging of cartilage in the long-term follow-up after Perthes disease. J Pediatr Orthop. 2010;30(2):147-53.

29. Orfanos I, Magkou C, Anastasopoulos I, Karanikas E, Sitaras NM. Urine glycosaminoglycans in children with transient hip synovitis and Perthes disease. J Pediatr Orthop B. 2005;14(2):92-6.

30. Eckerwall $G$, Lohmander LS, Wingstrand $H$. Increased levels of proteoglycan fragments and stromelysin in hip joint fluid in Legg-Calvé-Perthes disease. J Pediatr Orthop. 1997;17(2):266-9.

31. Zreiqat H, Howlett CR, Gronthos S, Hume D, Geczy CL. S100A8/S100A9 and their association with cartilage and bone. J Mol Histol. 2007;38(5):381-91.

32. Barksby HE, Hui W, Wappler I, Peters HH, Milner JM, Richards CD, et al. Interleukin-1 in combination with oncostatin M up-regulates multiple genesin chondrocytes:implications for cartilage destruction and repair. Arthritis Rheum. 2006;54:540-50.

33. Chen F, Kerner MB, Dorfman HD, Hamerman D. The distribution of S-100 protein in articular cartilage from osteoarthritic joints. J Rheumatol. 1990;17(12):1676-81.

34. Schelbergen RF, Blom AB, van den Bosch MH, Slöetjes A, Abdollahi-Roodsaz S, Schreurs BW, et al. Alarmins S100A8 and S100A9 elicit a catabolic effect in human osteoarthritic chondrocytes that is dependent on Toll-like receptor 4. Arthritis Rheum. 2012;64(5):1477-87.

35. Hammer HB, Odegard S, Fagerhol MK, Landewé $R$, van der Heijde D, Uhlig T, et al. Calprotectin (a major leucocyte protein) is strongly and independently correlated with joint inflammation and damage in rheumatoid arthritis. Ann Rheum Dis. 2007;66(8):1093-7.

36. Brun JG, Jonsson R, Haga HJ. Measurement of plasma calprotectin as an indicator of arthritis and disease activity in patients with inflammatory rheumatic diseases. J Rheumatol. 1994;21(4):733-8.

37. Hirata T, Fujioka M, Takahashi KA, Arai Y, Asano T, Ishida M, et al. ApoB C7623T polymorphism predicts risk for steroid-induced osteonecrosis of the femoral head after renal transplantation. J Orthop Sci. 2007;12(3):199-206.

38. Wang XY, Niu XH, Chen WH, Lin N, Song JN, Chen B, et al. [Effects of apolipoprotein $\mathrm{A} 1$ and $\mathrm{B}$ gene polymorphism on avascular necrosis of the femoral head in Chinese population]. Zhongguo Gu Shang. 2008;21(2):99-102.

39. Jones Jr JP. Fat embolism and osteonecrosis. Orthop Clin North Am. 1985;16(4):595-633.

40. Rappaport EB, Fife D. Slipped capital femoral epiphysis in growth hormone-deficient patients. Am J Dis Child. 1985;139(4):396-9.

41. Kitoh H, Kitakoji T, Kawasumi M, Ishiguro N. A histological and ultrastructural study of the iliac crest apophysis in Legg-Calve-Perthes disease. J Pediatr Orthop. 2008;28(4):435-9.

42. He W, Li K. Incidence of genetic polymorphisms involved in lipid metabolism among Chinese patients with osteonecrosis of the femoral head. Acta Orthop. 2009;80(3):325-9.

\section{Submit your next manuscript to BioMed Central and take full advantage of:}

- Convenient online submission

- Thorough peer review

- No space constraints or color figure charges

- Immediate publication on acceptance

- Inclusion in PubMed, CAS, Scopus and Google Scholar

- Research which is freely available for redistribution 